\title{
In situ Probing of Structural and Electromechanical Properties of an Individual ZnO Nanobelt
}

Anjana Asthana*, Kasra Momeni*, Abhishek Prasad**, Yoke Khin Yap**, Reza Shahbazian Yassar*. *Department of Mechanical Engineering- Engineering Mechanics, Michigan Technological University, Houghton, MI 49931

**Department of Physics, Michigan Technological University, Houghton, MI 49931

Nanogenerators [1], piezoelectric field effect transistors [2] and piezoelectric diodes [3] were recently developed based on the unique coupling of piezoelectric and semiconducting properties of $\mathrm{ZnO}$ nanowires. The emergence of this nanopeizotronic area [4] requires further understanding on the electromechanical behavior of $\mathrm{ZnO}$ nanostructures. Although there were a few reports on the electromechanical behavior of $\mathrm{ZnO}$ nanowires [5], no studies are devoted on the $\mathrm{ZnO}$ nanobelts, which are structurally different. Here, we present the study on the electrical and structural properties of an individual nanobelt using atomic force microscopy (AFM) and in situ high resolution transmission electron microscopy (TEM). All the measurements were carried out on a single tilt AFM-TEM holder (Nanofactory Instruments) in a JEM 4000FX TEM system that operated at 200kV. Figs. 1(a-c) display the images of a typical $\mathrm{ZnO}$ nanobelt undergoing a sequential stress by incremental movement of the peizodriven tungsten tip towards the AFM tip. A series of measured $I-V$ curves with an increase of stress in $\mathrm{ZnO}$ nanobelt are respectively shown in Fig. 1d.

With the increase in stress, the current is dramatically increased with start off voltage of $5.5 \mathrm{~V}$ bias (curve "c" in Fig. 2d) and shows the semiconducting behavior. In a large bias regime, the $I-V$ curve can be differentiated to obtain a resistance $\mathrm{R}$ of the nanobelt $(R \sim \mathrm{d} V / \mathrm{d} I$,$) . We found that for this$ compressed state, the resistance of the nanobelt was decreased to $33 \mathrm{M} \Omega$ from $\sim 80 \mathrm{M} \Omega$ in the lightly stressed state shown in Fig. $1 \mathrm{~b}$. The nonlinear $I-V$ characteristics of these stressed states suggests for a semiconducting behavior. Thus our measurement system can be regarded as a metal-semiconductor-metal $(M-S-M)$ circuit [6]. The related semiconducting parameters can be retrieved from the experimental $I-V$ data in the bias regime $>5 \mathrm{~V}$, by the following relation [7]. Here $S$ is the contact area associated with a bias; $J_{s}$ is slowly varying function of the applied bias. Based on this procedure, the resistance, resistivity, carrier concentration and carrier mobility were extracted as summarized in Table 1.

In conclusion, we have shown that the electrical transport properties of the nanobelt could be tuned from the insulating to semiconducting by inducing stress into the nanobelt using an in situ AFM-TEM stage. The semiconducting parameters were retrieved from the experimental $I-V$ curves using the $M-S-M$ model. The structural investigations of the nanobelts show that each nanobelt is single crystalline with hexagonal wurtize structure and grown in the [0110] direction. 


\section{References:}

[1] Z. L. Wang and J. H. Song, Science 312 (2006) 242.

[2] X. D. Wang, J. Zhou, J. H. Song, J. Liu, N. S. Xu, and Z. L. Wang, Nano lett. 6 (2006) 2768.

[3] J. H. He, C. L. Hsin, J. Liu, L. J. Chen and Z. L. Wang, Adv. Mater.( Weinheim, Ger.) 19(2007) 781. [4] Z. L. Wang, Adv. Mater. (Weinheim, Ger.) 19, 889 (2007).

[5] K. H. Liu, P. Gao, Z. Xu, X. D. Bai and E. G. Wang, APL 92 (2008) 213105.

[6] F. A. Padovani and R. Stratton, Solid-State Electron. 9, (1966) 695.

[7] Z. Y. Zhang, C. H. Jin, X. L. Liang, Q. Chen and L. -M. Peng, APL. 88 (2006) 73102.

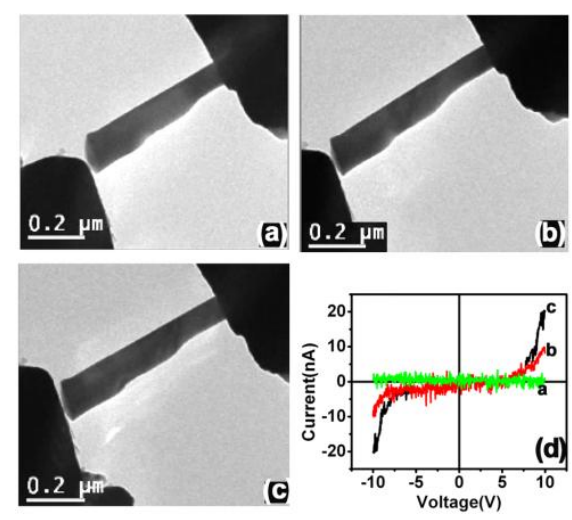

\begin{tabular}{|lcc|}
\hline \multicolumn{3}{|c|}{ Electrical paramters of Zno nano belts } \\
& Curve b & Curve c \\
Resistance $(\mathrm{M} \Omega):$ & 80 & 33 \\
Resistivity $(\Omega \mathrm{cm}):$ & 21.98 & 10.36 \\
$\mathrm{E}_{0}(\mathrm{meV}):$ & 25.9 & 26.4 \\
Carrier concentration $/(\mathrm{cm} 3):$ & $1.1 \times 10^{17}$ & $2.5 \times 10^{17}$ \\
Mobilit $\left(\mathrm{cm}^{2} / \mathrm{Vs}\right)$ & 4.45 & 2.41 \\
\hline
\end{tabular}

